\title{
Synthesis and mechanism of aluminum silicate mesoporous materials by F108 template
}

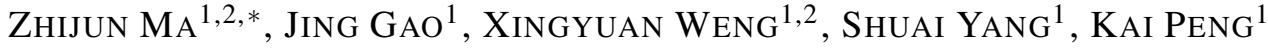 \\ ${ }^{1}$ College of Mining, Liaoning Technical University, Fuxin 123000, China \\ ${ }^{2}$ Resarch Center of Coal Resources Safe Mining and Clean Utilization, LNTU, China
}

\begin{abstract}
Aluminosilicate mesoporous materials were synthesized using F108 template (polyethylene glycol-polypropylene glycolpolyethylene glycol) at a concentration of $0.034 \mathrm{~g} / \mathrm{ml}$ and the molar ratio of $\mathrm{Al}_{2} \mathrm{O}_{3}$ to $\mathrm{SiO}_{2}$ was 0.09 . The products were then characterized using nitrogen adsorption/desorption tests, scanning electron microscopy (SEM), and X-ray diffraction (XRD). The effects of various crystallization temperatures as well as sodium hydroxide concentrations on the average diameter, pore volume, surface area, and morphology of the synthetic material were analyzed. Results showed that a sodium hydroxide concentration of $11 \mathrm{~mol} / \mathrm{L}$ and a crystallization temperature of $130{ }^{\circ} \mathrm{C}$ produced a synthetic material with regular pore size and homogeneous arrangement including a specific surface area of $137.62 \mathrm{~m}^{2} / \mathrm{g}$, an average pore volume of $0.27 \mathrm{~cm}^{3} / \mathrm{g}$, along with an average pore size of $15.33 \mathrm{~nm}$.
\end{abstract}

Keywords: template; hydrothermal synthesis; aluminosilicate; mesoporous materials; crystallization temperature

\section{Introduction}

Porous materials have been divided into three categories by the International Union of Pure and Applied Chemistry (IUPAC) according to the size of hole diameter. They include microporous materials (aperture less than $2 \mathrm{~nm}$ ), macroporous materials (pore size greater than $50 \mathrm{~nm}$ ), and mesoporous materials (aperture between $2 \mathrm{~nm}$ and $50 \mathrm{~nm}$ ) [1]. Mesoporous materials, in particular have proven to be favorable for use as catalysts in heterogeneous catalytic reactions of large molecules, giving these materials broad application prospects in the field of catalysis [2]. Compared with a zeolite molecular sieve, the porous walls of mesoporous silica lack both acidity and an ion exchange center that are usual requirements for the use in catalytic reactions. However, introduction of some metal elements (such as $\mathrm{Al}, \mathrm{Fe}, \mathrm{Mg}$, etc.) into synthetic silicate materials can effectively solve this problem as these additives can function as an acid center as well as a center for ion exchange. These modifications allow the material to be used directly as

*E-mail: zhijunma0930@126.com a catalyst for acid catalytic reactions [3-5]. Mesoporous materials can also be used in the field of environmental protection. Selective doping in ordered mesoporous materials can improve photoactivity and increase the efficiency of visible light catalytic degradation of organic wastes [6]. In the field of functional materials, MCM-41 mesoporous material can be used as an adsorbent to treat heavy metal ions (such as $\mathrm{Hg}, \mathrm{Ag}, \mathrm{Cr}, \mathrm{Pb}, \mathrm{Ba}, \mathrm{Zn}$, etc.) in wastewater due to the introduction of sulfurcontaining organic compounds on the inner surface of the pores [7].

Methods for the synthesis of mesoporous materials include microwave synthesis, room temperature synthesis, non-aqueous systems, and the most widely used synthesis method - hydrothermal synthesis [8-11]. Hydrothermal synthesis for mesoporous materials uses the formation conditions of natural zeolite minerals [12]. This method has the advantages of simplicity, high purity products, good dispersion, and ease of control of the particle size. In this work, we used a hydrothermal synthesis method to successfully synthesize aluminosilicate mesoporous materials using an F108 template to block the formation of copolymers. 
Furthermore, the effects of various sodium hydroxide concentrations and crystallization temperatures on product morphology, pore size, specific surface area, and pore volume were examined.

\section{Materials and methods}

Materials: F108 $\left(\mathrm{PEO}_{133}-\mathrm{PPO}_{50}-\mathrm{PEO}_{133}, \mathrm{AR}\right.$, SIGMA-ALDRICH), sodium metaaluminate $\left(\mathrm{NaAlO}_{2}, \mathrm{AR}\right.$, Sinopharm Chemical Reagent Co., Ltd.), water glass $\left(\mathrm{Na}_{2} \mathrm{O} \cdot \mathrm{nSiO}_{2}\right.$, Industrial Products, Shenyang North Chemical Plant), sodium hydroxide $(\mathrm{NaOH}, \mathrm{AR}$, Tianjin Komiou Chemical Reagent Development Center), concentrated hydrochloric acid $(\mathrm{HCl}, \mathrm{AR}$, Beijing Chemical Factory), absolute ethanol $\left(\mathrm{CH}_{3} \mathrm{CH}_{2} \mathrm{OH}, \mathrm{AR}\right.$, Liaoning Quanrui Century Co., Ltd.).

Instruments: The specific surface area, pore volume and pore size of the composite material were tested at a liquid nitrogen temperature $(77 \mathrm{~K})$ using an Autosorb-iQ automatic surface area and pore size distribution analyzer from Quantachrome Instruments U.S. The synthetic sample before the test needs to be vacuum evacuated at $573 \mathrm{~K}$ for $10 \mathrm{~h}$. The specific surface area of the sample was calculated by the Brunauer-Emmett-Teller (BET) method, and the specific surface area was calculated based on the adsorption data between $\mathrm{P} / \mathrm{P}_{0}=0.05-0.3$ :

$$
A_{S}=\left(V_{m} / 2214\right) N_{A} \sigma
$$

where $\mathrm{V}_{\mathrm{m}}$ is the volume of the monolayer; 2214 is the molar volume of the gas; $\mathrm{N}_{\mathrm{A}}$ is the Avogadro constant; for the area covered by each adsorbate molecule, the nitrogen molecule is generally $0.612 \mathrm{~nm}^{2}$.

The average pore size and pore volume were calculated according to the Barrett-Joyner-Halenda (BJH) model, in which the pore volume is calculated using the adsorption amount at a relative pressure $\mathrm{P} / \mathrm{P}_{0}=0.99$.

$\mathrm{X}$-ray diffraction (XRD) patterns were measured by a Shimadzu XRD-6100 diffractometer using $\mathrm{CuK} \alpha$ radiation with a light tube voltage of $40 \mathrm{kV}$, a current of $30 \mathrm{~mA}$, and a scan rate of $0.5^{\circ} / \mathrm{min}$ and $10^{\circ} / \mathrm{min}$ in the test ranges of $0.5^{\circ}$ to $5^{\circ}$ and $5^{\circ}$ to $90^{\circ}$.
A field emission scanning electron microscope, model XL-30, produced by FEI, USA, was used to observe the morphology of the sample. A small sample was placed in a centrifuge tube containing an appropriate amount of absolute ethanol, and shaken for $1 \mathrm{~h}$ in a CQ-50 ultrasonic cleaner. The dispersed sample was dropped on a square silicon wafer with a side length of about $1 \mathrm{~cm}$, and the sample was sprayed with gold. The morphology of the sample was directly observed under a scanning electron microscope.

Treatment of the template: $2.4 \mathrm{~g}$ of F108 template was completely dissolved in $70 \mathrm{~cm}^{3}$ of distilled water using an ultrasonic cleaner for $1 \mathrm{~h}$. Composition of the mesoporous material guide agent: $3 \mathrm{~g}$ of sodium aluminate was dissolved in $30 \mathrm{~cm}^{3}$ portion of sodium hydroxide solution of various concentrations. $70 \mathrm{~cm}^{3}$ of waterglass was then added dropwise and allowed to react for $1 \mathrm{~h}$ at $25{ }^{\circ} \mathrm{C}$.

Preparation of mesoporous material: $70 \mathrm{~cm}^{3}$ of template agent was added to each of four flasks and kept in a water bath at $35{ }^{\circ} \mathrm{C}$. The solution was stirred at $100 \mathrm{rpm}$ while $100 \mathrm{~cm}^{3}$ of directing agent was added and mixed until obtaining uniform consistency. Hydrochloric acid was added dropwise in order to regulate the $\mathrm{pH}$ level and maintain a white emulsion. After continued stirring for $2 \mathrm{~h}$, the reaction solution was transferred to a Teflonlined autoclave serving as the crystallization reactor and placed in an oven to crystallize for $60 \mathrm{~h}$. The crystallized product was then washed, filtered, and dried at $550{ }^{\circ} \mathrm{C}$ in a calcination furnace for $6 \mathrm{~h}$. An overview of the experimental process is shown in Fig. 1.

\section{Results and discussion}

\subsection{X- ray diffraction (XRD) analysis}

Fig. 2 shows the X-ray diffraction patterns collected for mesoporous materials crystallized at $110{ }^{\circ} \mathrm{C}$ for $60 \mathrm{~h}$ using different concentrations of sodium hydroxide. Fig. 3 shows X-ray diffraction patterns collected for mesoporous silica synthesized using a sodium hydroxide concentration of $11 \mathrm{~mol} / \mathrm{L}$ and crystallized for $60 \mathrm{~h}$ at various temperatures. 


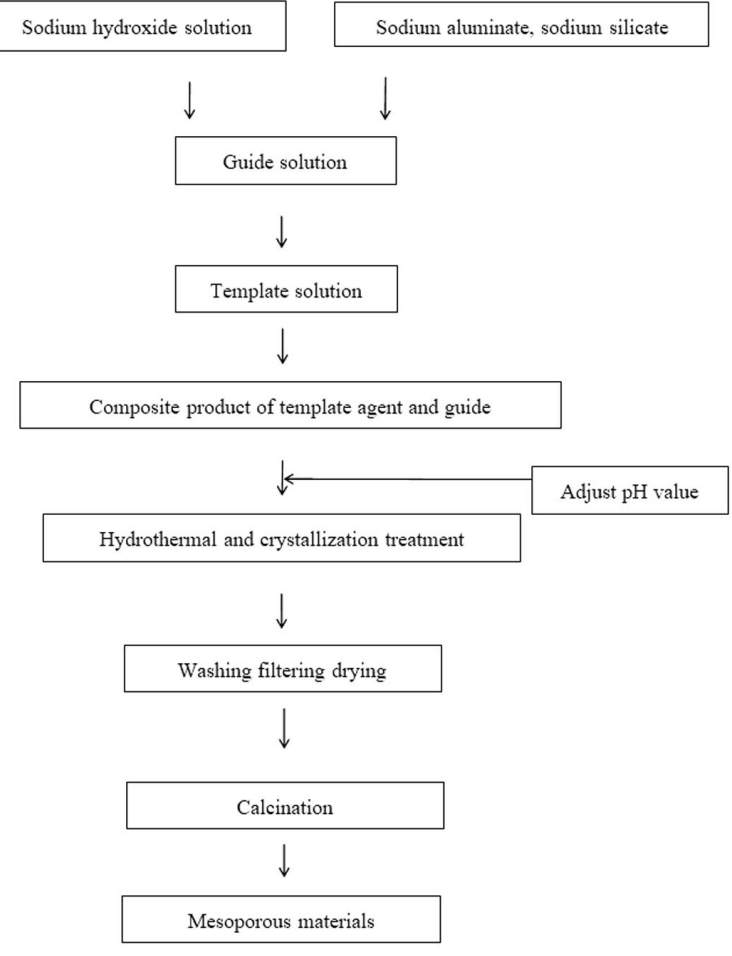

Fig. 1. An overview of the experimental process.

As shown in Fig. 2 and Fig. 3, aluminosilicate mesoporous materials synthesized using different concentrations of sodium hydroxide and crystallization temperatures do not show any obvious Bragg diffraction peaks indicative of long-range order structure. However, at $23^{\circ}$ a small dispersion in the width of the peak is seen, indicating that shortrange order still exists. Based on these results it can be inferred that the synthetic material is amorphous.

\subsection{Scanning electron microscope (SEM) analysis}

Scanning electron microscopy (SEM) images of mesoporous materials synthesized using various concentrations of sodium hydroxide and crystallized at $110{ }^{\circ} \mathrm{C}$ for $60 \mathrm{~h}$ are shown in Fig. 4. In this figure, all of the mesoporous materials synthesized using different concentrations of sodium hydroxide lack an ordered structure and show irregular channels. Comparing Fig. 4a and Fig. 4b, it is obvious that increasing the concentration of sodium hydroxide also increased the porosity

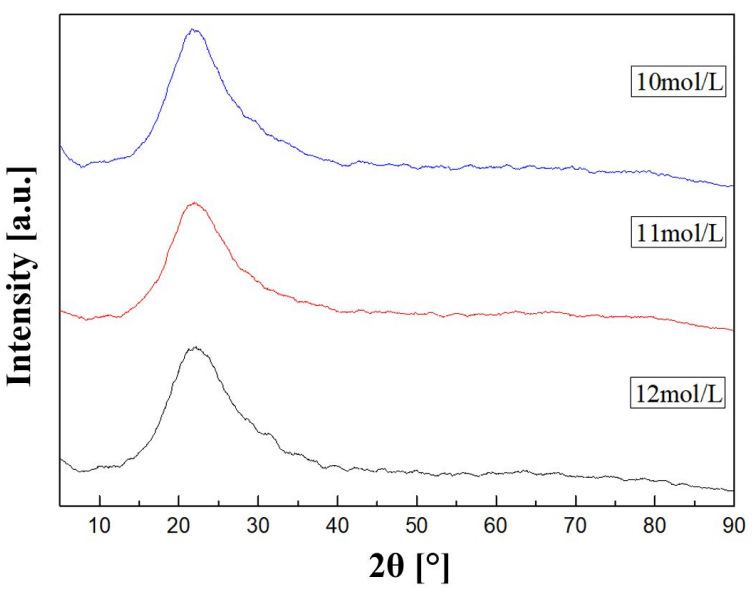

Fig. 2. X-ray diffraction patterns of aluminosilicate mesoporous materials synthesized using various concentrations of sodium hydroxide.

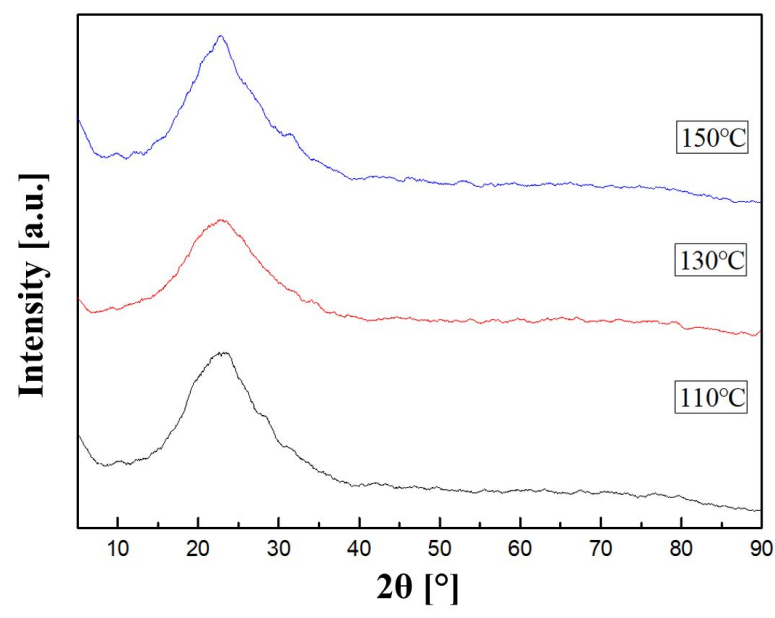

Fig. 3. X-ray diffraction patterns of aluminosilicate mesoporous materials synthesized at different crystallization temperatures.

of the mesoporous material. This is most likely due to the fact that as the $\mathrm{Na}^{+}$concentration increased, the critical micelle temperature for the F108 template agent decreased, which hindered crystallization into micelles with regular and orderly arrangement. Instead, synthetic growth continued with a uniform distribution of materials. The distribution of pore size of the sample shown in Fig. $4 \mathrm{c}$ is extremely irregular, and is probably due to the high concentration of sodium hydroxide leading to destruction of F108 template micelles. 


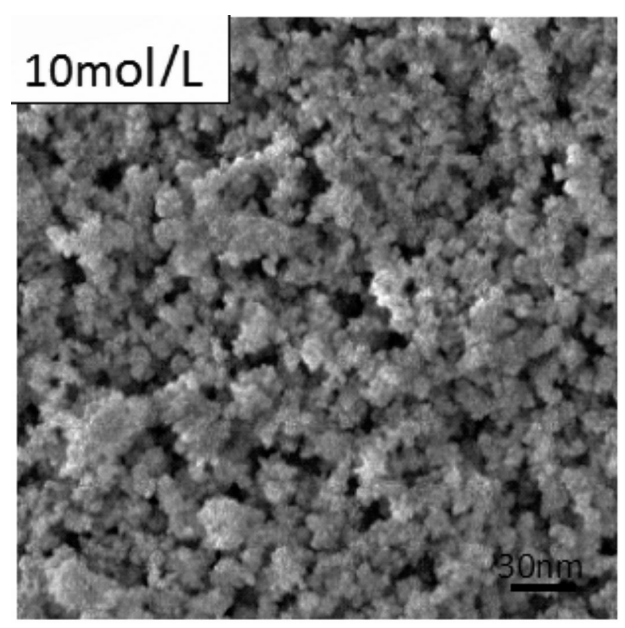

(a)

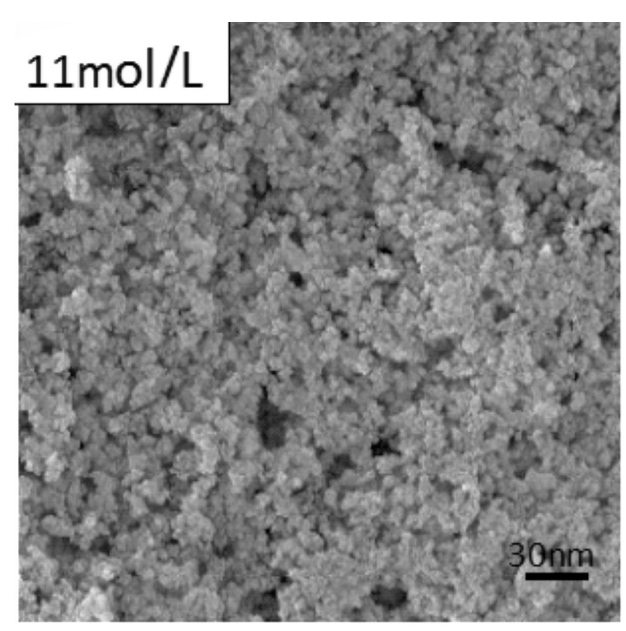

(b)

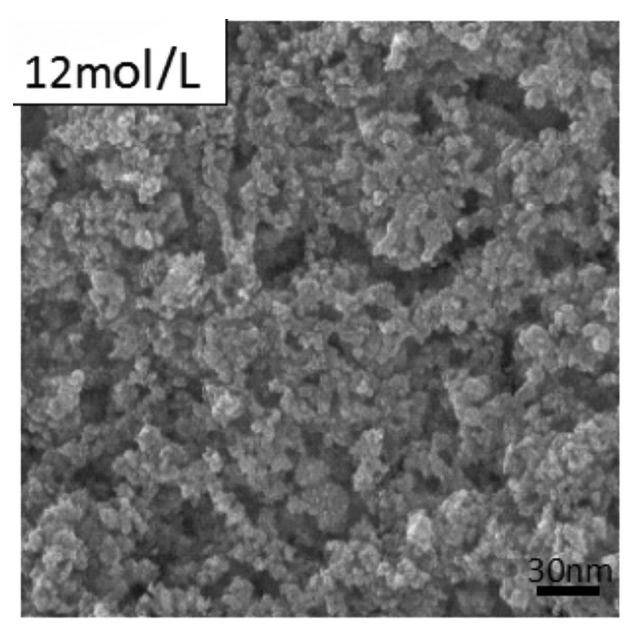

(c)

Fig. 4. Scanning electron microscope images of aluminosilicate mesoporous materials synthesized using different concentrations of sodium hydroxide.
Using a sodium hydroxide concentration of $11 \mathrm{~mol} / \mathrm{L}$ and a crystallization time of $60 \mathrm{~h}$, synthesis of aluminosilicate mesoporous material was completed at different crystallization temperatures and examined using scanning electron microscopy (SEM), as shown in Fig. 5. In Fig. 5, no long-range ordered structure exists for the mesoporous materials synthesized at different crystallization temperatures. However, short-range ordered structure is visible, and is especially apparent in Fig. 5b These results are consistent with the conclusions reached due to the analysis of XRD data. Fig. 5a shows the synthesized material having fine and irregular pores, which may be due to the low crystallization temperature. At low temperatures, the F108 template was unable to reach the critical micelle formation temperature, leading to messy, singlestranded F108 micelles. Fig. 5c shows wedgeshaped hole material, flake particle accumulation, and both large and thin channels. These structures may have formed due to the fact that the crystallization temperature exceeded the critical temperature of the F108 template which led to chaotic results. In contrast, only the pore size and arrangement seen in Fig. 5b appear relatively neat. Therefore, this may be a good crystallization temperature for the F108 template, in that it leads to the formation of a neat and orderly arrangement of the micelles.

\subsection{Nitrogen adsorption-desorption analysis}

At a crystallization temperature of $110{ }^{\circ} \mathrm{C}$ and crystallization time of $60 \mathrm{~h}$, silicon aluminate mesoporous materials were synthesized using different concentrations of sodium hydroxide and characterized using nitrogen adsorption and desorption, as shown in Fig. 6. In this figure, the adsorption-desorption isotherms of two samples (Fig. 6a and Fig. 6b) were found to be of type IV, with a turning point at $\mathrm{P} / \mathrm{P}_{0}=0.2$, and monolayer adsorption occurring before that turning point. Furthermore, there is an obvious capillary condensation phenomenon occurring at $\mathrm{P} / \mathrm{P}_{0}=0.5$, indicative of the existence of mesoporous material with pore sizes around $5 \mathrm{~nm}$ or so. Fig. $6 \mathrm{c}$ shows type III $\mathrm{N}_{2}$ adsorption-desorption isotherms, where the 


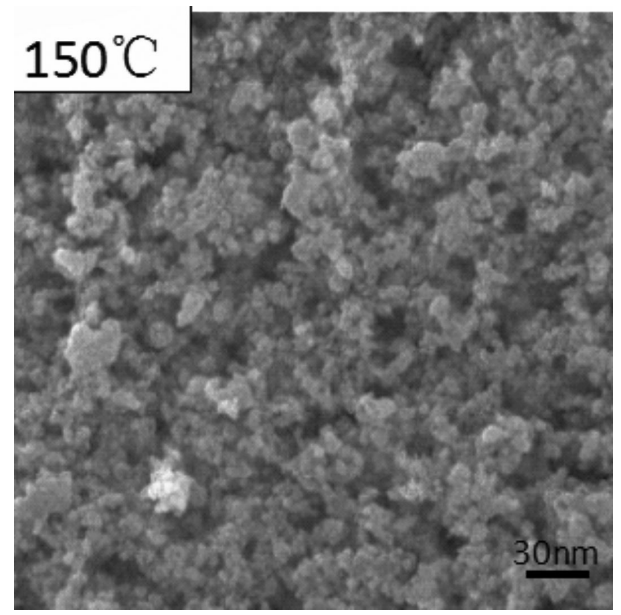

(a)

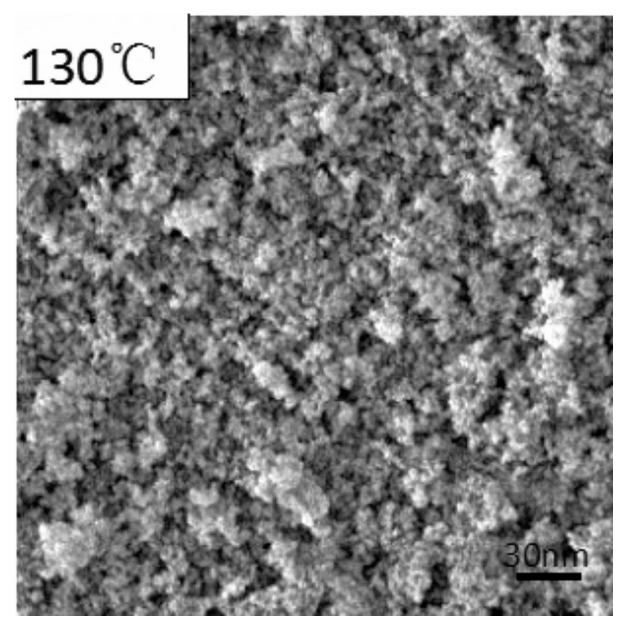

(b)

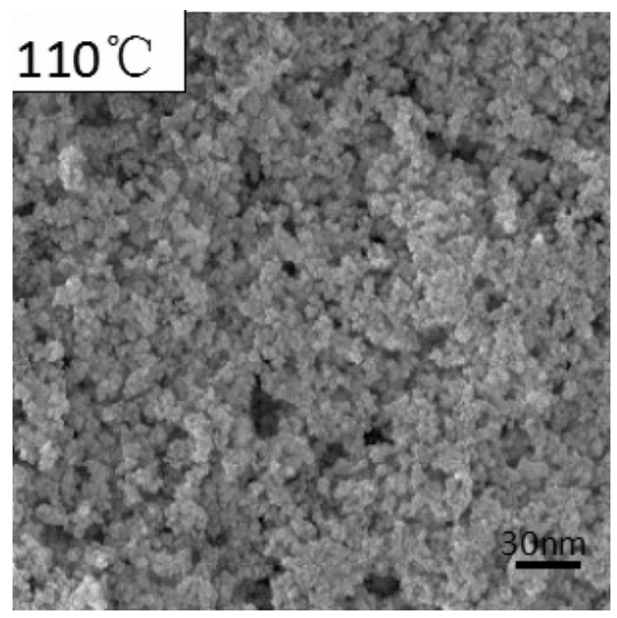

(c)

Fig. 5. Scanning electron microscope images of aluminosilicate mesoporous material synthesized at different crystallization temperatures. relative pressure $\mathrm{P} / \mathrm{P}_{0}<0.7$ is concave downward. This is due to the fact that the interactions of the synthesized mesoporous material with $\mathrm{N}_{2}$ are less than the interactions between $\mathrm{N}_{2}$ and $\mathrm{N}_{2}$, specifically in the first layers of the sample where both the heat of $\mathrm{N}_{2}$ adsorption and the heat of condensation are small. When $0.7<\mathrm{P} / \mathrm{P}_{0}<1.0$, the adsorption equilibrium leads to the growth of $\mathrm{H}_{3}$ hysteresis loop, with structure present in a sample for more mesoporous and macroporous parts, including the accumulation of flaky particles as well as slit pore formation. As the relative pressure $\left(\mathrm{P} / \mathrm{P}_{0}\right)$ rises to around 1, the adsorption layer is large enough for capillary and pore condensation phenomena to occur. The amount of adsorption increases sharply due to the larger size distribution (5 $\mathrm{nm}$ to $30 \mathrm{~nm}$ ). Large holes still exist, so the high pressure side of the isotherm is in a saturated state.

Table 1 shows the specific surface area (calculated using the BET model) as well as pore volume and average pore size (calculated using the BJH model) of silicon aluminate mesoporous materials synthesized at crystallization temperature of $110{ }^{\circ} \mathrm{C}$ and crystallization time of $60 \mathrm{~h}$, with different concentrations of sodium hydroxide. From Table 1, we can see that as the concentration of sodium hydroxide was increased, the specific surface area of the sample decreased while the average pore size increased. When the concentration of sodium hydroxide was $12 \mathrm{~mol} / \mathrm{L}$, the specific surface area of the sample was quite small. This may be due to the low porosity leading to particle formation in the slit apertures. When the sodium hydroxide concentration was lower than $11 \mathrm{~mol} / \mathrm{L}$, the specific surface area was relatively larger and the average pore size was smaller. This is most likely due to the smaller pore size and higher porosity of the synthetic material. Based on these results, the optimal concentration of alkali for the synthesis of this mesoporous material is $11 \mathrm{~mol} / \mathrm{L}$.

Using a sodium hydroxide concentration of $11 \mathrm{~mol} / \mathrm{L}$ and a crystallization time of $60 \mathrm{~h}$, aluminate mesoporous materials were synthesized at different crystallization temperatures and were then characterized using nitrogen adsorption and desorption, as shown in Fig. 7. Fig. 7a shows the same pattern as described and analyzed in Fig. $7 b$. 
Table 1. Structural parameters of samples prepared using different concentrations of sodium hydroxide.

\begin{tabular}{cccc}
\hline $\begin{array}{c}\text { Sodium hydroxide } \\
\text { concentration }[\mathrm{mol} / \mathrm{L}]\end{array}$ & $\begin{array}{c}\text { Specific surface area } \\
{\left[\mathrm{m}^{2} / \mathrm{g}\right]}\end{array}$ & $\begin{array}{c}\text { Pore volume } \\
{\left[\mathrm{cm}^{3} / \mathrm{g}\right]}\end{array}$ & $\begin{array}{c}\text { Average pore diameter } \\
{[\mathrm{nm}]}\end{array}$ \\
\hline \hline 10 & 297.55 & 0.24 & 4.68 \\
11 & 239.75 & 0.25 & 6.47 \\
12 & 45.02 & 0.18 & 27.50 \\
\hline
\end{tabular}

Fig. 7c shows type III results in the area of low pressure $\mathrm{P} / \mathrm{P}_{0}<0.3$, with little adsorption and no inflection point, indicating that the force between the sample and the $\mathrm{N}_{2}$ was very weak. With an increase of pressure, the adsorption capacity increased significantly, specifically when $\mathrm{P} / \mathrm{P}_{0}>0.8$. At these pressures an obvious capillary condensation phenomenon is apparent, indicating the presence of mesopores with larger pore size around $20 \mathrm{~nm}$. Fig. 7b results are a combination of type III and type IV isotherms. When $\mathrm{P} / \mathrm{P}_{0}>0.7$, an obvious capillary condensation phenomenon is apparent, indicating the presence of mesopores with larger pore size around $15 \mathrm{~nm}$. For all three samples, values of $\mathrm{P} / \mathrm{P}_{0}$ between 0.5 and 1 show an $\mathrm{H}_{3}$ hysteresis loop, a common feature for mesoporous materials that have a small amount of large holes usually with lamellar particles that have formed from the accumulation of a woven type hole. The hysteresis loop closed at $\mathrm{P} / \mathrm{P}_{0}=0.4$, showing that there are also smaller features in the mesoporous material, with apertures around $2 \mathrm{~nm}$ to $3 \mathrm{~nm}$.

Table 2 shows the specific surface area calculated using the BET model as well as the pore volume and average pore size calculated using $\mathrm{BJH}$ for the silicon aluminate mesoporous material under different crystallization conditions. As shown in Table 2, with an increase in the temperature of crystallization, the specific surface area and pore volume of the material first increase then decrease. When the crystallization temperature was lower than $130{ }^{\circ} \mathrm{C}$, the specific surface area was relatively large and the average pore size was relatively small, due to the small pore size and high porosity of the composite material. When the crystallization temperature was higher than $130{ }^{\circ} \mathrm{C}$, the opposite was true; the material showed a smaller specific surface area and larger pore size. This may be due to accumulation of lamellar particles forming larger and less porous wedge shaped holes. It is also possible that these effects were due to collapse of the material caused by the high crystallization temperature. These results in combination with the nitrogen adsorption - desorption isotherm analysis showed that when the crystallization temperature was $130{ }^{\circ} \mathrm{C}$, the pore size of synthetic material was around $15 \mathrm{~nm}$ and had a relatively uniform distribution. In this condition, the specific surface area reached $137.62 \mathrm{~m}^{2} / \mathrm{g}$, the pore volume was $0.27 \mathrm{~cm}^{3} / \mathrm{g}$, and average pore size was $15.33 \mathrm{~nm}$.

\subsection{Mechanism of micelle formation by the template}

The formation of surfactant micelles ultimately determines the morphology of synthetic aluminosilicate mesoporous materials, including aperture size and other characteristics. For this reason understanding the micelle formation mechanism by the template is necessary.

Generally it is believed that micelle formation is due to "hydrophobic interactions", meaning that attractive non-polar molecules between the non-polar groups lead to the association of these groups in the aqueous environment [13]. When the surface active agent was dissolved in water, the original hydrogen bonding structure of the water was destroyed. The disrupted water molecules formed a more ordered structure by surrounding the added polar organic molecules, leading to an overall reduction in the entropy of the system and therefore an unfavorable entropy change. To restore the entropy of the system, surface active agents aggregate nearby to form micelles, restoring water hydrogen bonding structure in the solution. Micelles align to form a molecular chain due to hydrophobic 
Table 2. Structural parameters of samples prepared at different crystallization temperatures.

\begin{tabular}{cccc}
\hline $\begin{array}{c}\text { Crystallization temperature } \\
{\left[{ }^{\circ} \mathrm{C}\right]}\end{array}$ & $\begin{array}{c}\text { Specific surface area } \\
{\left[\mathrm{m}^{2} / \mathrm{g}\right]}\end{array}$ & $\begin{array}{c}\text { Pore volume } \\
{\left[\mathrm{cm}^{3} / \mathrm{g}\right]}\end{array}$ & $\begin{array}{c}\text { Average pore diameter } \\
{[\mathrm{nm}]}\end{array}$ \\
\hline \hline 110 & 239.75 & 0.25 & 6.47 \\
130 & 137.62 & 0.27 & 15.33 \\
150 & 81.60 & 0.26 & 16.73 \\
\hline
\end{tabular}

interactions. The micelle formation process in aqueous solution is driven primarily by entropy, as the enthalpy is weak. The hydrophobic effect, along with its effect on the entropy of the system increases with an increase in temperature. This may be due to the elevated temperature, leaving water hydrogen bonding intact while destroying the hydrogen bonds along the more hydrophobic surfaces, which results in overall weakening of the hydrophobic effect. Because the PEO-PPO-PEO block copolymer was free in the aqueous solution, the free energy change of the system during the micellization process was negative. This means that the thermodynamically stable micelles formed spontaneously, and that the change in standard enthalpy for the micelle formation was positive. Furthermore, the transfer of single strands from the solution to micelles was an endothermic process and unfavorable in terms of enthalpy. Therefore, it can be concluded that the increase of entropy is the driving force for the formation of block copolymer micelles [14].

\subsection{Adsorption theory of surfactant on solid-liquid interface}

The adsorption of surfactants on solid-liquid interface has important applications in many industrial productions. There are many types of adsorption isotherms, and many experimental results have been reported. The adsorption of a single surfactant on the solid-liquid interface can be divided into three categories, Langmuir, S and LS.

\subsubsection{Adsorption theory}

There are two stages of surfactants adsorption on the solid-liquid interface. The first stage is the adsorption of individual surfactant molecules or ions by electrostatic attraction and direct interaction between van der Waals gravity and solid surface:

$$
\begin{aligned}
& \text { Adsorption potential + Monomer } \\
& \text { = Adsorbed monomer }
\end{aligned}
$$

Monomer refers to an individual surface active molecule or ion, the equilibrium constant of which is:

$$
K_{1}=a_{1 / a_{s}} a
$$

where a is the activity of the monomer in solution, and the dilute solution can be replaced by the concentrated monomer; $\mathrm{a}_{1}$ and $\mathrm{a}_{s}$ are the activity of the adsorbed monomer and the empty adsorption site, respectively. In the second stage, surface active molecules or ions form surface micelles by hydrophobic interaction between hydrocarbon chains, This is the active center of the first stage of the adsorption monomer forming the surface micelles. When there is balance:

$$
\begin{aligned}
& (n-1) \text { Monomer }+ \text { Adsorbed monomer } \\
& =\text { Surface micelles }
\end{aligned}
$$

The equilibrium constant is:

$$
K_{2}=a_{h m /} a_{1} a^{n-1}
$$

where $a_{\mathrm{hm}}$ is the activity of the surface micelles, and $\mathrm{n}$ is the aggregation number of the surface micelles.

Approximately said, $\mathrm{a}_{1}, \mathrm{a}_{\mathrm{hm}}$, and $\mathrm{a}_{\mathrm{s}}$ can be replaced by monomer adsorption capacity $\tau_{1}$, surface micelle adsorption capacity $\tau_{h m}$ and adsorption site number $\tau_{\mathrm{s}}$, respectively. Thus formulas 3 and 5 can be written as 6 and 7, respectively.

$$
K_{1}=\tau_{1} / \tau_{s} c
$$




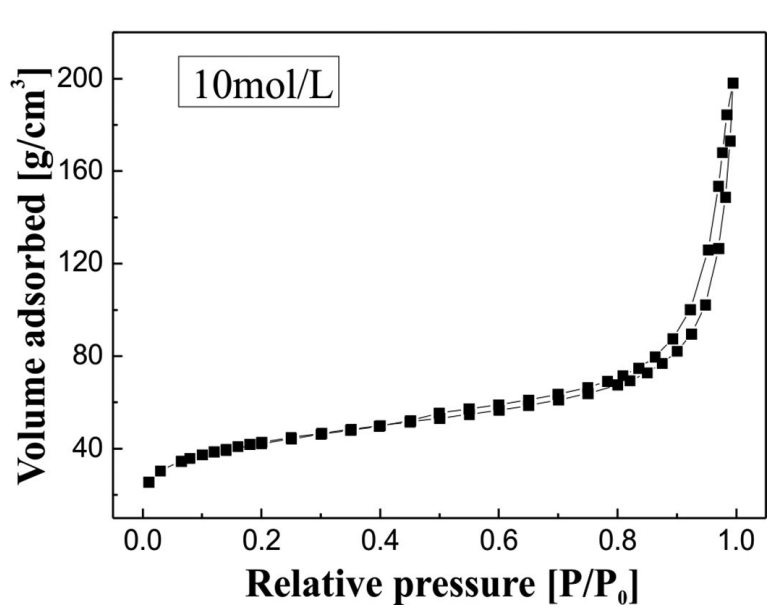

(a)

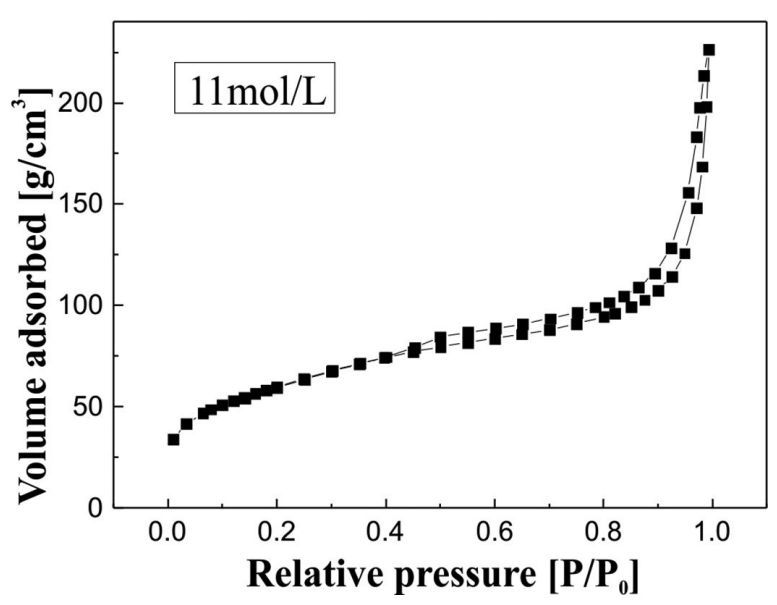

(b)

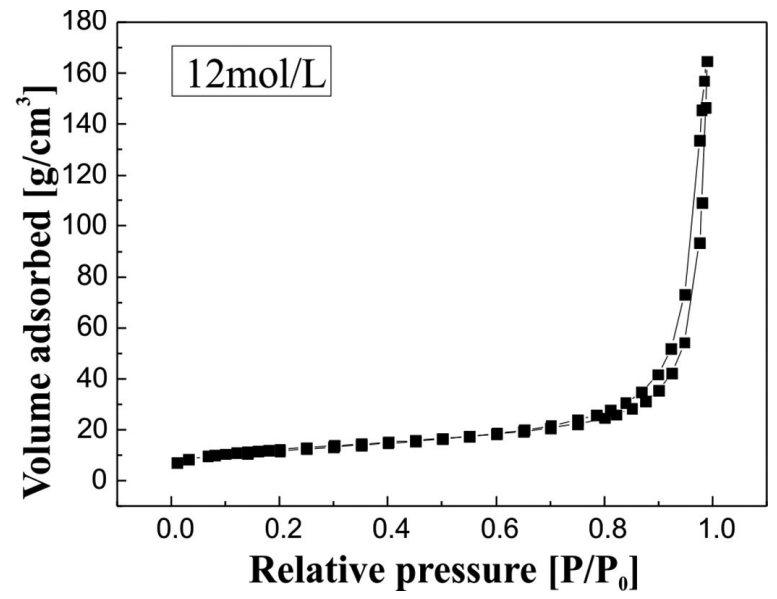

(c)

Fig. 6. Nitrogen adsorption-desorption isotherms of aluminosilicate mesoporous materials synthesized using different concentrations of sodium hydroxide.

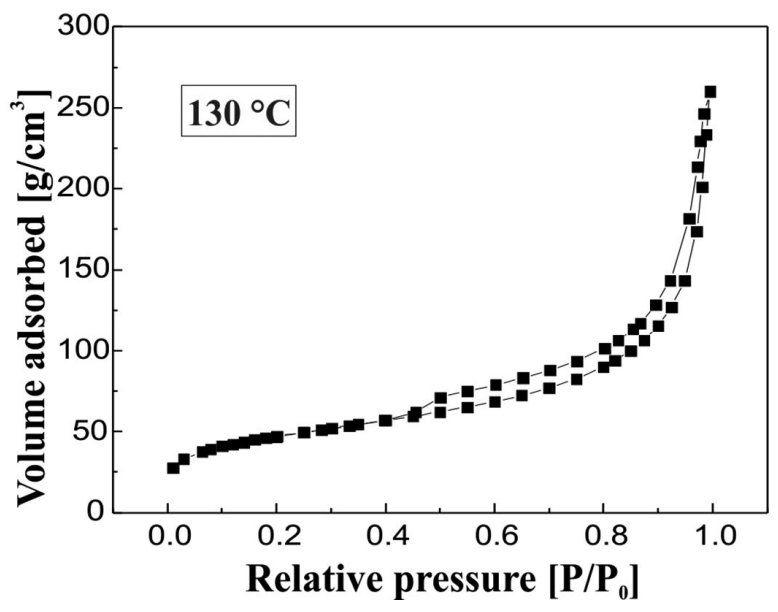

(a)

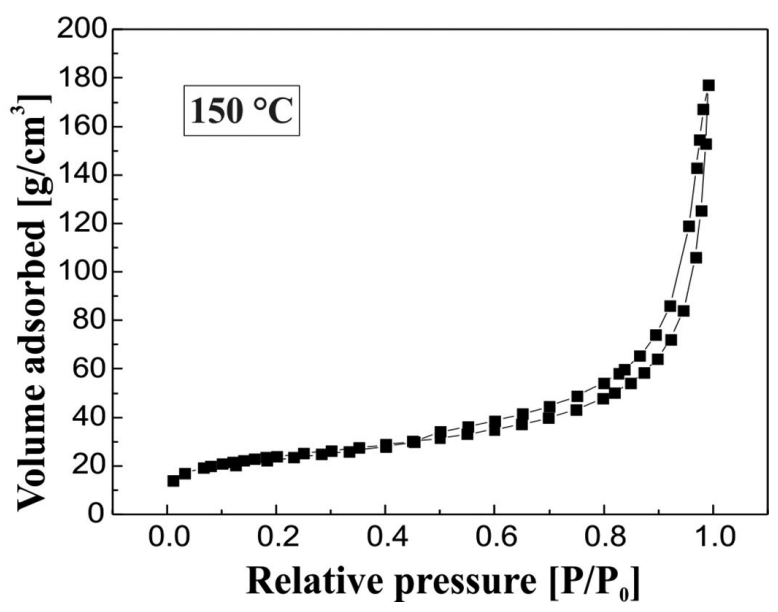

(b)

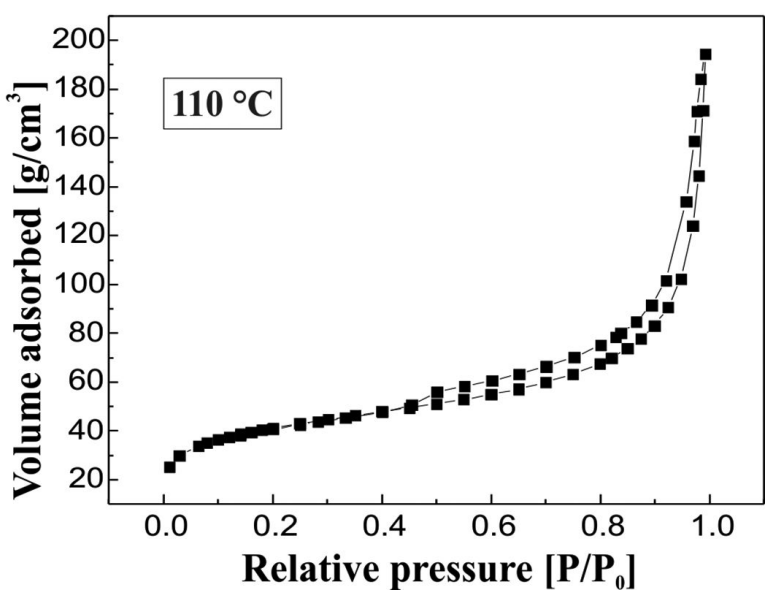

(c)

Fig. 7. Nitrogen adsorption-desorption isotherms of mesoporous synthetic silicon crystallized at different temperatures. 


$$
K_{2}=\tau_{h m} / \tau_{1} c^{n-1}
$$

According to the physical meaning of total adsorption capacity $\tau$ and saturated total adsorption capacity $\tau_{\infty}$ (at high concentration) at any concentration of $\mathrm{C}$, it can be obtained:

$$
\begin{gathered}
\tau=\tau_{1}+n \tau_{h m} \\
\tau_{\infty}=n \tau_{s}+\tau_{1}+n \tau_{h m}
\end{gathered}
$$

Both $\tau$ and $\tau_{\infty}$ can be measured experimentally. The general formula of adsorption isotherm can be derived by combining the formula $6,7,8$, and 9 .

$$
\tau=\frac{\tau_{\infty} k_{1} C\left(\frac{1}{n}+k_{2} C^{n-1}\right)}{1+k_{1} C\left(1+k_{2} C^{n-1}\right)}
$$

There are two important limit cases in equation 7 , when $\mathrm{k}_{2} \rightarrow 0, n \rightarrow 0$, formula 10 reduces to Langmuir formula 11 .

$$
\tau=\frac{\tau_{\infty} k_{1} C}{1+k_{1} C}
$$

If $\mathrm{n}>1$, formula 8 has two limit cases, when $\mathrm{k}_{2} \mathrm{C}^{n-1} \ll \frac{1}{n}$, formula 10 becomes 12 .

$$
\tau=\frac{\left(\tau_{\infty} / n\right) k_{1} C}{1+k_{1} C}
$$

Equation 10 is still Langmuir type, but the adsorption capacity of the single molecular limit is not $\tau_{\infty}$, but $\tau_{\infty} / \mathrm{n}$; when $\mathrm{k}_{2} \mathrm{C}^{\mathrm{n}-1} \gg 1, \mathrm{k}_{1} \mathrm{C} \ll 1, \mathrm{kC}^{\mathrm{n}}$, equation 10 can be transformed into equation 13 .

$$
\tau=\frac{\tau_{\infty} k C^{n}}{1+k C^{n}}
$$

Among them, we have combined the one-step formation model of surface micelles with the law of mass action, and we have derived the formula 13 . When the concentration is increasing, both the formula 10 and the formula 13 are reduced to formula 14 .

$$
\tau=\tau_{\infty}
$$

\subsubsection{Calculation of adsorption theory for- mula}

When $\mathrm{k}_{2}=10^{25}, \mathrm{n}=8, \mathrm{k}_{1}$ takes different values, then according to the adsorption isotherm curve calculated according to formula 10 , the micellar concentration of surfactant at the calculation is $10^{-3} \mathrm{~mol} / \mathrm{dm}^{3}$. The calculated data are shown in Table 3 and in Fig. 9. The curves a to f from left to right correspond to $\mathrm{k}_{1}=\left(10^{5}, 10^{4}, 10^{3}, 10^{2}, 10,1\right)$.

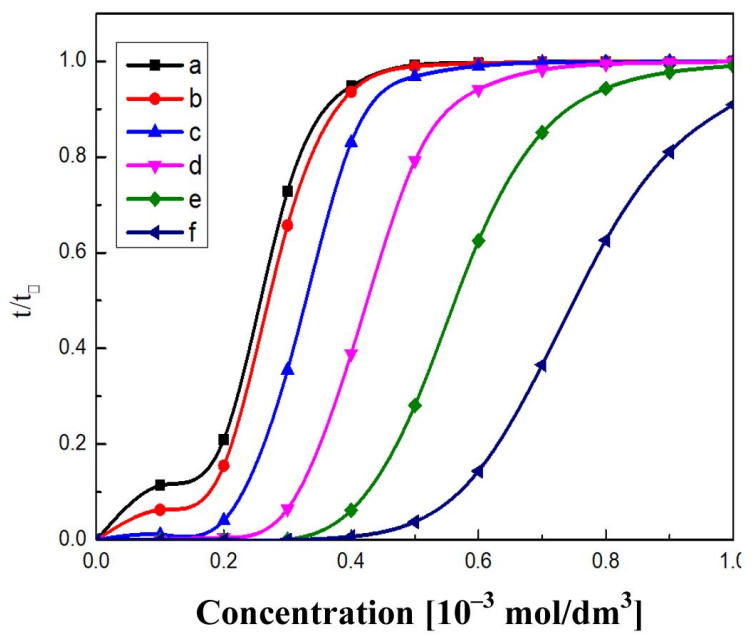

Fig. 8. Adsorption isotherms for different $\mathrm{k}_{1}$ ( $\mathrm{a}-\mathrm{f}$ show $\mathrm{k}_{1}$ of the order $\left.10^{5}, 10^{4}, 10^{3}, 10^{2}, 10,1\right)$.

Fig. 8 shows the influence of concentration on $\tau / \tau_{\infty}$, at different $k_{1}$ values. Obviously, when $k_{1}$ is large, the LS isotherm can be obtained, and when $\mathrm{k}$ is very small, the equation can be transformed into the isothermal curve of $\mathrm{S}$ type, which is the combined isotherm of type III and type IV of the Langmuir isotherm, respectively. The results are consistent with the $\mathrm{N}_{2}$ adsorption and desorption isotherms of the final synthesized materials, which indicates that the silica and aluminum sources are uniformly distributed on the micelles, and the mesoporous materials are obtained by calcining the micelles to remove the surfactant micelles. The $\mathrm{N}_{2}$ adsorption and desorption isotherm of the final synthesized material is shown in Fig. 9. 
Table 3. Value of $\tau / \tau_{\infty}$ for different $k_{1}$.

\begin{tabular}{ccccccc}
\hline $\mathrm{C} / 10^{-3} \mathrm{~mol} / \mathrm{dm}^{3}$ & \multicolumn{7}{c}{ Value of $\mathrm{k}_{1}$ at different time $\tau / \tau_{\infty}$} \\
& $10^{5}$ & $10^{4}$ & $10^{3}$ & $10^{2}$ & $10^{1}$ & 1 \\
\hline \hline 0.0 & 0 & 0 & 0 & 0 & 0 & 0 \\
0.1 & 0.11 & 0.06 & 0.01 & 0 & 0 & 0 \\
0.2 & 0.21 & 0.15 & 0.04 & 0 & 0 & 0 \\
0.3 & 0.73 & 0.66 & 0.35 & 0.06 & 0.01 & 0 \\
0.4 & 0.94 & 0.93 & 0.83 & 0.38 & 0.06 & 0 \\
0.5 & 0.99 & 0.98 & 0.96 & 0.79 & 0.28 & 0.03 \\
0.6 & 0.99 & 0.99 & 0.99 & 0.94 & 0.62 & 0.14 \\
0.7 & 1.00 & 0.99 & 0.99 & 0.98 & 0.85 & 0.36 \\
0.8 & 1.00 & 1.00 & 0.99 & 0.99 & 0.94 & 0.62 \\
0.9 & 1.00 & 1.00 & 1.00 & 0.99 & 0.97 & 0.81 \\
1.0 & 1,00 & 1.00 & 1.00 & 1.00 & 0.99 & 0.91 \\
\hline
\end{tabular}

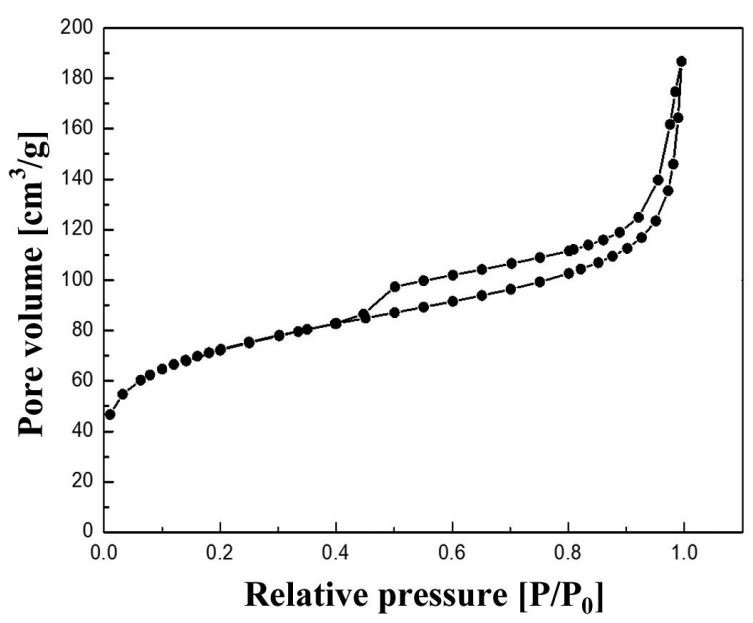

Fig. 9. Nitrogen adsorption-desorption isotherm of the final synthesized material.

\section{Conclusion}

Silicon aluminate mesoporous materials were synthesized using $0.034 \mathrm{~g} / \mathrm{mL}$ F108 template agent and the molar ratios of $\mathrm{Al}_{2} \mathrm{O}_{3}$ and $\mathrm{SiO}_{2}$ of 0.09 . The agents were placed in a $35^{\circ} \mathrm{C}$ water bath and stirred at $160 \mathrm{rpm}$. The crystallization reaction was performed at various temperatures for $60 \mathrm{~h}$ using different $\mathrm{NaOH}$ concentrations. The product was then washed, filtered by suction, dried, and calcined and the resulting product showed an amorphous structure with no long-range order structure, but some short-range ordered structures.
The sodium hydroxide concentration and crystallization temperature greatly influence the synthesis of the aluminosilicate mesoporous materials. It shouldn't be too high or too low. The use of an appropriate crystallization temperature and correct ratio of sodium hydroxide to F108 template agent leads to the formation of neat and orderly micelles. From this work, the optimum synthesis parameters for creating mesoporous materials with uniform pore size distribution were determined. The parameters: sodium hydroxide concentration of $11 \mathrm{~mol} / \mathrm{L}$ and crystallization temperature of $130{ }^{\circ} \mathrm{C}$ are the optimal. Use of these conditions led to the formation of materials with a specific surface area of $137.62 \mathrm{~m}^{2} / \mathrm{g}$, an average pore volume of the $0.27 \mathrm{~cm}^{3} / \mathrm{g}$, and an average pore size of $15.33 \mathrm{~nm}$.

\section{Acknowledgements}

Authors ZHIJUN MA and JING GAO contributed equally to this work. The project was supported by the Natural Science Foundation of China (51372108) and the Research Center of Coal Resources Safe Mining and Clean Utilization, LNTU, China.

\section{References}

[1] IUPA C., Pure Appl. Chem., 603 (1985), 619.

[2] PEI D.Y., Dong Y.Z., DAVD I.M., Nature, 152 (1998), 155.

[3] Luan Z., Cheng C., Klinowsk J., J. Phys. Chem., 1018 (1995), 1024

[4] Biz S., White M.G., J. Phys. Chem., 8432 (1999), 8442 . 
[5] LiN Y., LiN H., Mou C., Micropor. Mesopor. Mater., 203 (2004), 208.

[6] Hu H.J., ZhaO H.Q., WANG L.Z., Mineral Prot. Util., 10 (1998), 13.

[7] Song C., Yong R.Y., Kui X.Z., Jin D.W., Catal. Today, 116 (2006), 2.

[8] LU B., Gui Z.N., Yi H.W., Deng H., GaO F.Z., YAN F.Z., MENG L.W., YU H.S., Micropor. Mesopor. Mater., 54 (2016), 58.

[9] Pranjal K., Choitayna D.P., Prantu D., J. Mol. Catal. A: Chem., 145 (2014), 150.

[10] Joanna G., Anna O., Izabela N., Micha M., Robert P., Chem. Eng. J., 209 (2016), 219.
[11] Xiao B.W, Min Y.S, Bo M., Nai T.Y., Xiao Y.A., Mater. Lett., 59 (2016), 62.

[12] Lee N.K., Hammad R.K., Electrochim. Acta., 22 (2016), 30.

[13] Chandler D., Nature, 640 (2005), 647.

[14] Alexandridisl P., HolzWARTH J.F., Hatton T.A., J. Macromol., 2414 (1994), 2425.
Received 2018-07-02 Accepted 2019-04-23 